\title{
Assessment of Plasmodium antigens and CRP in dried blood spots with multiplex malaria array
}

\author{
Ihn Kyung Jang ${ }^{1}\left(\mathbb{D} \cdot\right.$ Sara Aranda $^{1} \cdot$ Rebecca Barney $^{1} \cdot$ Andrew Rashid $^{1} \cdot$ \\ Muhammad Helwany ${ }^{1}$ John C. Rek ${ }^{2}$ Emmanuel Arinaitwe ${ }^{2}$ - Harriet Adrama ${ }^{2}$. \\ Maxwell Murphy ${ }^{3}$ - Mallika Imwong ${ }^{4}$ - Stephane Proux ${ }^{5}$ - Warat Haohankhunnatham ${ }^{5}$. \\ Xavier C. Ding ${ }^{6}$. François Nosten ${ }^{5,7} \cdot$ Bryan Greenhouse $^{3} \cdot$ Dionicia Gamboa $^{8}$. \\ Gonzalo J. Domingo ${ }^{1}$
}

Received: 7 July 2020/ Accepted: 21 November 2020/Published online: 3 January 2021

(C) The Author(s) 2021

\begin{abstract}
Dried blood spots (DBS) typically prepared on filter papers are an ideal sample type for malaria surveillance by offering easy and cost-effective methods in terms of sample collection, storage, and transport. The objective of this study was to evaluate the applicability of DBS with a commercial multiplex malaria assay, developed to concurrently measure Plasmodium antigens, histidine-rich protein 2 (HRP2), Plasmodium lactate dehydrogenase $(\mathrm{pLDH})$, and a host inflammatory biomarker, C-reactive
\end{abstract}

Supplementary Information The online version of this article ( https://doi.org/10.1007/s12639-020-01325-2) contains supplementary material, which is available to authorized users.

Ihn Kyung Jang

ikjang@path.org

1 Diagnostics Program, PATH, Seattle, WA, USA

2 Infectious Diseases Research Collaboration, Kampala, Uganda

3 Department of Medicine, University of California at San Francisco, San Francisco, CA, USA

4 Faculty of Tropical Medicine, Department of Molecular Tropical Medicine and Genetics, Mahidol University, Bangkok, Thailand

5 Faculty of Tropical Medicine, Mahidol-Oxford Tropical Medicine Research Unit, Shoklo Malaria Research Unit, Mahidol University, Mae Sot, Thailand

6 The Foundation for Innovative New Diagnostics, Geneva, Switzerland

7 Nuffield Department of Medicine, Centre for Tropical Medicine and Global Health, University of Oxford, Oxford, UK

8 Departamento de Ciencias Celulares y Moleculares, Facultad de Ciencias y Filosofía, Instituto de Medicina Tropical Alexander von Humboldt, Universidad Peruana Cayetano Heredia, Lima, Peru protein (CRP), in whole blood. The assay conditions were optimized for DBS, and thermal stability for measurement of Plasmodium antigens and CRP in dried blood were determined. Performance of the multiplex assay on matched DBS and whole blood pellet samples was also evaluated using the clinical samples. The results indicate the acceptable performance in multiplex antigen detection using DBS samples. At cutoff levels for DBS, with a diagnostic specificity with a lower 95\% confidence bound $>92 \%$, diagnostic sensitivities against polymerase chain reaction (PCR)-confirmed malaria for HRP2, Pf LDH, $P v$ LDH, and Pan LDH were 93.5\%, 80.4\%, 21.3\%, and $55.6 \%$, respectively. The half-life of pLDH was significantly less than that of HRP2 in thermal stability studies. Results with DBS samples collected from Peru indicate that the uncontrolled storage conditions of DBS can result in inaccurate reporting for infection with $P$. falciparum parasites with $h r p 2 / 3$ deletions. With careful consideration that minimizing the unfavorable DBS storage environment is essential for ensuring integrity of heat-labile Plasmodium antigens, DBS samples can be used as an alternative to liquid whole blood to detect $P$. falciparum with $h r p 2 / 3$ deletions in malaria surveillance.

Keywords Malaria $\cdot$ HRP2 $\cdot$ LDH $\cdot$ Multiplex ·

Immunoassay $\cdot$ Dried blood spot

\section{Introduction}

Malaria is currently estimated to infect around 219 million individuals, resulting in 435,000 deaths each year (World Health Organization 2018). The World Health Organization aims to reduce malaria case incidence and mortality rates by at least $90 \%$ by 2030 . Two human Plasmodium 
species, $P$. falciparum and $P$. vivax, cause major clinical complications, with $P$. falciparum leading to a higher mortality. Asymptomatic infections pose a problem for malaria elimination as the infected asymptomatic individuals may serve as reservoirs of parasites for continuous malaria transmission (Bousema et al. 2014; Chen et al. 2016). A significant proportion of asymptomatic infections are undetectable by microscopy and can only be detected by highly sensitive nucleic acid amplification or antigen detection assays for malaria parasite proteins (Slater et al. 2019; Jang et al. 2019; Plucinski et al. 2019; Wu et al. 2015).

P. falciparum histidine-rich protein 2 (HRP2) is a primary protein biomarker for malaria diagnosis. A highly sensitive rapid diagnostic test (RDT) targeting HRP2 has shown mixed results in the improvement of identifying low-level blood stage P. falciparum infection (Das et al. 2017; Landier et al. 2018; Das et al. 2018). All HRP2based RDTs, which recognize a homolog of HRP2, HRP3 as well and are most widely used in Africa, can give false negative results when applied to infections with $P$. falciparum parasites with a partial or complete deletion of hrp 2 and hrp3 (Pati et al. 2018; Kumar et al. 2013). Given these restrictions associated with HRP2, RDTs identifying $P$. falciparum with hrp2/3 deletions and other Plasmodium species are needed in areas where infections with $h r p 2 / 3$ deletion mutants and other species are prevalent. Other biomarkers like Plasmodium lactate dehydrogenase (pLDH) and Plasmodium aldolase (pAldo) have been targeted in malaria RDTs (Jain et al. 2014). Especially, pLDH carrying both species-specific and Pan-specific epitopes can allow identification of these $h r p 2 / 3$ deletion mutants of $P$. falciparum and differentiate two major species and all Plasmodium species (Hurdayal et al. 2010).

Quantitative laboratory immunoassays for Plasmodium antigens have been developed on three multiplexed platforms: the simultaneous capture and sequential detection (SCSD) assay (Markwalter et al. 2016), the Luminex beadbased assay (Rogier et al. 2017; Martianez-Vendrell et al. 2020), and enzyme-linked immunosorbent assay (ELISA)based assay using Q-Plex ${ }^{\mathrm{TM}}$ technology (Jang et al. 2019). The SCSD assay detects Pan LDH and HRP2, and the Luminex bead-based assay detects pan aldolase, Pan LDH, and HRP2. The Q-Plex ${ }^{\mathrm{TM}}$ Human Malaria Array (5-Plex, Quansys Biosciences, Logan, Utah), simultaneously measures HRP2, $P f$ LDH, $P v$ LDH, Pan LDH, and CRP (Jang et al. 2020). These multiplexed assays have shown potential for screening for $P$. falciparum with $h r p 2 / 3$ deletions and the improved detection of P. vivax (Jang et al. 2019). Performance of the Human Malaria Array 5-Plex to date has only been demonstrated on whole blood specimens.

Whole blood samples present multiple challenges to large-scale field survey studies, including inconvenient sample collection, storage, and transport that can subsequently increase the overall cost and the accidental biohazardous risks during sample collection and transport. On the other hand, dried blood spot (DBS) samples, which are generally prepared from a finger-prick onto filter paper and subsequently dried, address these problems and are routinely collected in malaria epidemiological research studies. DBS-derived samples have been applied to assays measuring parasite nucleic acids to map the spread of drugresistant malaria parasites and as serological markers to measure malaria transmission intensity (Pearce et al. 2003; Goodhew et al. 2014; Plucinski et al. 2018). In this study, we evaluate the use of DBS as an additional sample type for determining malaria infection via antigen detection using the Human Malaria Array 5-Plex assay. We compared assay performance with matched DBS and whole blood pellet samples from malaria-infected individuals obtained from diverse sources. We also investigated the temporal and thermal stability of each Plasmodium antigen and CRP on DBS stored under a range of different temperatures for up to 240 days.

\section{Materials and methods}

\section{Ethics}

One hundred and forty de-identified whole blood samples of asymptomatic individuals from Myanmar and Uganda were collected under informed consent and with institutional review board (IRB) approvals as described previously (Das et al. 2017). Thirty-two de-identified $P$. falciparum clinical DBS samples of symptomatic individuals were obtained at two different study sites in Peru with informed consent and with IRB approval from Universidad Peruana Cayetano Heredia (UPCH, Lima, Peru; UPCH 52707) (Gamboa et al. 2010).

\section{Plasmodium sample preparation for validation}

To prepare DBS, frozen venous ethylenediaminetetraacetic acid (EDTA) blood samples collected from two study sites, Uganda and Myanmar, were thawed and used for spotting $60 \mu \mathrm{l}$ blood per marked circle on Whatman 903 Protein saver cards (Cytiva [formerly GE Healthcare Life Sciences], Marlborough, MA) in the PATH laboratory. Care was taken to avoid touching the paper with the pipette tip by allowing the drop of blood to hang from the tip of the pipette so as to mimic DBS collection from a fingerstick blood droplet. Each card was dried overnight at room temperature $\left(23{ }^{\circ} \mathrm{C}\right)$ at ambient humidity (approximately $30 \%$ ) and packaged individually in Mylar bags with silica gel desiccant and humidity indicator cards (Desiccare, Inc., 
Las Vegas, NV). Recombinant HRP2 and CRP proteins were purchased from Microcoat Biotechnologie $\mathrm{GmbH}$ (Bernried am Starnberger See, Germany) and HyTest (Turku, Finland), respectively. Recombinant $P f$ LDH and $P v$ LDH proteins were purchased from MyBioSource (San Diego, CA). These recombinant malaria proteins and CRP were spiked into SeraCare ${ }^{\mathrm{TM}}$ Basematrix processed plasma (Fisher Scientific) mixed with washed red blood cells at a ratio of 6 to 4 from malaria-negative human blood purchased from PlasmaLab International (Everett, WA) to generate matched whole blood pellets and DBS samples at different protein concentrations. Malaria-negative human blood $(>10 \mathrm{~mL}$ ) was centrifuged at $1,000 \mathrm{~g}$ for $10 \mathrm{~min}$ and washed twice with PBS. Some whole blood and DBS samples were also prepared by spiking $P$. falciparum laboratory strain W2 (BEI Resources, Manassas, VA)-infected red blood cells from in vitro culture into a malaria-negative blood pool. With the exception of DBS that were introduced into the stability test, all DBS were stored at $-20{ }^{\circ} \mathrm{C}$ and $<30 \%$ relative humidity and whole blood samples were stored at $-80{ }^{\circ} \mathrm{C}$ until use.

\section{P. falciparum samples with hrp2/3 deletions from UPCH}

Sample collection and storage condition for the Peru samples have been described previously for the determination of the limit of detection with the best performing HRP2-based RDTs (Gamboa et al. 2010). Finger-prick blood samples were collected on Whatman filter paper. At the time of collection, parasitemia was determined by thick smear microscopy. DBS samples were stored at $-20{ }^{\circ} \mathrm{C}$. De-identified DBS samples from two different studies conducted in 2007-2008 (Foundation for Innovative New Diagnostics [FIND] 2007, FIND 2008) and in 2014 (FIND 2014) were made available for assessment of HRP2, pLDH, and CRP using the 5-Plex. Genomic DNA isolated from DBS samples were characterized for identification of $P$. falciparum infection and genotyping of $h r p 2$ and $h r p 3$ by PCR (Gamboa et al. 2010).

\section{Evaluation of target analytes in DBS and whole blood}

All patient DBS and whole blood samples were tested blinded to PCR results. For optimization of DBS assays, elution buffer and elution procedures involving incubation time and the addition of a shaking step were evaluated. A 6-mm diameter disc was punched out from the DBS with a sterile standard one-hole paper punch. Each dried blood disc was transferred into one well in a low-binding V-bottom 96-well plate (Thermo Scientific) with calibrator diluent (Quansys Bioscience, Logan, UT) using sterile forceps. The amount of whole blood present in one punch was estimated to be $15 \mu \mathrm{L}$ on the basis of calculation of the applied blood volume, the measured size of the blood spread, and the assumption that the analytes are homogenous throughout the blood spot. The plate containing the blood spots was incubated overnight in $4{ }^{\circ} \mathrm{C}$ and then gently shaken at $500 \mathrm{rpm}$ for $1 \mathrm{~h}$ at room temperature. Calibrator was reconstituted in Calibrator diluent, and 7-point dilution and blank samples were prepared for Calibration curve. The eluates were tested in two replicates on the 5-Plex using a slightly modified protocol of mixing the eluates in $4 \times$ concentrated competitor mix to reduce the sample dilution instead of using $1 \times$ competitor mix which contains the biotin-labeled CRP competitor for the competitive CRP assay. Whole blood pellet samples were tested in a single replicate on the 5-Plex after mixing with $1 \times$ competitor mix (1:4). After addition of $50 \mu \mathrm{l}$ of Calibrators and samples, the plate was incubated at room temperature with shaking at $500 \mathrm{rpm}$ for $2 \mathrm{~h}$. Plates were then washed with wash buffer three times. A 50- $\mu$ l aliquot of detection mix was added to each well, and the plate was incubated with shaking for another hour and then washed again. For detection, a $50-\mu l$ aliquot of horseradish peroxide (HRP)-conjugated streptavidin solution was added to each well and then incubated with shaking for $30 \mathrm{~min}$. After a final wash, a $50-\mu l$ aliquot of chemiluminescent substrate solution was added to each well and the chemiluminescent intensity from the array spots in each well was measured using the Q-View Imager Pro (Quansys Biosciences) at an exposure time of $300 \mathrm{~s}$.

\section{Thermal stability experiment}

Whatman 903 Protein saver cards spotted with spiked whole blood samples containing recombinant HRP2, Pf $\mathrm{LDH}$ and $P v \mathrm{LDH}$ proteins, and CRP were dried overnight on the bench at $<30 \%$ humidity. Then, the dried cards were stored in Mylar bags containing the desiccant and held at the following temperatures: $50{ }^{\circ} \mathrm{C}, 30^{\circ} \mathrm{C}$, room temperature, $4{ }^{\circ} \mathrm{C}$, and $-20^{\circ} \mathrm{C}$. Samples were stored up to 240 days. At varying time points, DBS were removed from storage, and the eluates from rehydrated DBS punches were analyzed using the 5-Plex. Percentage recovery of each biomarker was calculated relative to concentration of the controls measured in reference DBS samples stored at $20{ }^{\circ} \mathrm{C}$.

\section{Data analysis}

Biomarker concentrations in DBS eluates were interpolated from the standard curve using a 4-parameter logistic curvefit (Q-view imager software, Quansys Biosciences). To analyze data from the assay optimization, corrected 
concentrations of analytes in DBS samples were calculated from two replicate experiments by multiplication of the corresponding dilution factors $(3.25$ for the use of concentrated competitor and 10 for the volume of elution buffer). Results outside the limit of quantification were reported as above ( $>$ upper limit of quantification, ULOQ) or below the limit of quantification $(<$ lower limit of quantification, LLOQ) for each analyte. The raw concentration values from the Q-view report were used for data analysis, unless indicated otherwise. Intra-assay and interassay variations were assessed by calculating the coefficient of variation $(\mathrm{CV})$ for replicates of high, medium, and low control samples in a single plate, and 20 plates, respectively. Acceptance can be considered if $\mathrm{CV}$ values are less than $10 \%$ and $20 \%$ across three control samples for intra-assay and inter-assay precision, respectively (or the average of $\mathrm{CV}$ values for control samples is within those ranges). To assess the correlations between matched clinical whole blood and DBS samples, the Pearson correlation coefficient $(r)$ was estimated. The cutoff values in DBS assays, which are equivalent to those of blood assays, was calculated from the curve's equation obtained by means of linear regression analysis with data from matched clinical whole blood and DBS samples. The sensitivity and the specificity were calculated by the formulas (true positives)/ (true positives + false negatives) and (true negatives)/(true negatives + false positives), respectively. The half-lives $\left(\mathrm{t}_{1 / 2}\right)$ of biomarkers in DBS were assessed by monitoring the concentration of analytes over time with comparison to the first sample tested at each temperature. The half-life was calculated using a one phase exponential decay fit model (GraphPad Prism v.6, GraphPad Software, La Jolla, CA).

\section{Results}

\section{Optimization of elution conditions for DBS samples}

Procedures for extraction of malaria analytes and CRP from DBS samples were optimized by utilizing the calibrator diluent solution included in the 5-Plex kit. Variables for optimization included competitor concentration, volume of elution buffer, and a mix of elution temperature, shaking speed, and time. Increased extraction buffer volumes and more extended incubation time at room temperature led to significant improvement of recovery for all antigens throughout concentrations in five positive controls (S1, S2, S3, S4, and S5), whereas increased competitor concentration showed an effect only on the low concentration (Online Resource Figs. 1A, 1B, and 1C). Under the optimized assay procedure in which analytes are eluted from a DBS disc in $10 \times$ elution volume at $4{ }^{\circ} \mathrm{C}$ overnight followed by incubating the eluate/disc at room temperature for $1 \mathrm{~h}$ in shaking, and mixed in $4 \times$ competitor mix, the recovery of biomarkers in eluates of five control samples was examined as compared to matched blood control samples. The recovery from DBS of HRP2, Pf LDH, $P v$ LDH, and Pan LDH was 27\%, 24\%, 22\%, and $27 \%$, respectively, and $38 \%$ for CRP (Online Resource Table 1). There were excellent correlations between DBS and whole blood pellet measurements at $>0.96$ of $\mathrm{R}^{2}$ values for all biomarkers (Online Resource Fig. 2).

\section{DBS-based assay repeatability}

The assay variation was evaluated using positive DBS controls consisting of low, moderate, and high concentration of analytes in 20 replicates over multiple days or over a single day (Table 1). The results revealed that intra-assay average $\mathrm{CV}$ values for Plasmodium antigens met the acceptance criteria (below 10\%), at each analyte concentration (Table 1). Intra-assay average $\mathrm{CV}$ value for CRP was $10.7 \%$. For the inter-assay CVs the average CVs were all within the acceptance criteria (below 20\%) except for CRP, which was at $25.4 \%$. For Plasmodium antigens, only assays using the very low concentrations exceeded the $20 \%$ acceptance criteria (Table 1).

\section{Correlation between DBS and whole blood}

To evaluate whether antigen amounts in DBS eluates are correlated to paired whole blood samples, we measured the concentration of analytes in matched DBS and blood pellets from 140 clinical samples by the 5-Plex. The biomarker concentrations in the matched DBS and whole blood pellets showed good correlation with the following Pearson correlation coefficients: HRP2 $(r=0.951)$, Pf LDH $(r=0.931), \quad P v$ LDH $(r=0.811)$, Pan LDH $(r=0.923)$, and CRP $(r=0.716)$ (Fig. 1). Similar to results of five positive controls in DBS, the antigen concentration values in eluates from clinical DBS samples were lower than those in the corresponding whole blood pellet samples.

\section{Assessment of thermal stability of biomarker in DBS over time}

To evaluate the impact of temperature on the integrity of analytes, DBS samples were kept in a sealed bag with a desiccant package at different temperatures up to 240 days. The control samples stored at $-20{ }^{\circ} \mathrm{C}$ showed no change in antigen concentration over time as expected, whereas the biomarker concentrations in DBS stored at $4{ }^{\circ} \mathrm{C}$, room temperature, $30{ }^{\circ} \mathrm{C}$, and $50{ }^{\circ} \mathrm{C}$ decreased at increasing rates over the time (Fig. 2). The half-lives of the 
Table 1 Inter-assay and intra-assay variation of the 5-Plex testing dried blood samples. Assay variation of positive controls in dried blood $(n=20)$ were tested over multiple days and a single day. CVs
(\%) were calculated with concentration value of each analyte in DBS. Bolded are high CVs (\%) above the acceptance (20\% for inter-assay and $10 \%$ for intra-assay)

\begin{tabular}{|c|c|c|c|c|c|}
\hline \multicolumn{2}{|c|}{ Analyte calculated concentration $^{a}$} & \multicolumn{4}{|c|}{ Control ( $\mathrm{n}=20$ replicates $)$} \\
\hline & & Inter-assay CV (\%) & Average Inter-assay CV (\%) & Intra-assay CV (\%) & Average Intra-assay CV (\%) \\
\hline \multirow[t]{3}{*}{ HRP2 } & $268 \mathrm{pg} / \mathrm{mL}$ & 9.7 & 13.4 & 8.6 & 8.2 \\
\hline & $141 \mathrm{pg} / \mathrm{mL}$ & 10.3 & & 7.9 & \\
\hline & $12 \mathrm{pg} / \mathrm{mL}$ & 20.4 & & 8.2 & \\
\hline \multirow[t]{3}{*}{$P f \mathrm{LDH}$} & $2289 \mathrm{pg} / \mathrm{mL}$ & 7.9 & 14.3 & 7.8 & 8.0 \\
\hline & $1257 \mathrm{pg} / \mathrm{mL}$ & 10.9 & & 8.0 & \\
\hline & $113 \mathrm{pg} / \mathrm{mL}$ & 24.0 & & 8.4 & \\
\hline \multirow[t]{3}{*}{$P v \mathrm{LDH}$} & $793 \mathrm{pg} / \mathrm{mL}$ & 13.9 & 19.0 & 7.6 & 6.7 \\
\hline & $429 \mathrm{pg} / \mathrm{mL}$ & 12.7 & & 6.7 & \\
\hline & $37 \mathrm{pg} / \mathrm{mL}$ & 30.3 & & 5.8 & \\
\hline \multirow[t]{3}{*}{ Pan LDH } & $5033 \mathrm{pg} / \mathrm{mL}$ & 14.9 & 18.8 & 6.8 & 6.5 \\
\hline & $2752 \mathrm{pg} / \mathrm{mL}$ & 15.2 & & 5.7 & \\
\hline & $238 \mathrm{pg} / \mathrm{mL}$ & 26.4 & & 7.1 & \\
\hline \multirow[t]{3}{*}{ CRP } & $6190 \mathrm{ng} / \mathrm{mL}$ & 22.8 & 25.4 & 10.9 & 10.7 \\
\hline & $2776 \mathrm{ng} / \mathrm{mL}$ & 23.0 & & 8.5 & \\
\hline & $198 \mathrm{ng} / \mathrm{mL}$ & 30.6 & & 12.6 & \\
\hline
\end{tabular}

${ }^{\mathrm{a} C o n c e n t r a t i o n}$ values are uncorrected for dilution of analytes in eluates

biomarkers in DBS were calculated from non-linear regression models based on values in DBS stored at room temperature, $30{ }^{\circ} \mathrm{C}$, and $50{ }^{\circ} \mathrm{C}$, and storage time (Online Resource Table 2). All Plasmodium antigens exhibited longer half-life at lower temperature conditions, room temperature, and $30{ }^{\circ} \mathrm{C}$ than at $50{ }^{\circ} \mathrm{C}$. The most significant instability was observed with $\mathrm{pLDH}$ proteins as compared to HRP2 at each temperature condition. The CRP biomarker exhibited 88.3 days of half-life whereas Plasmodium antigens had a range between 12.9 and 21.6 days at $50{ }^{\circ} \mathrm{C}$. Overall results on the decay of biomarkers after 240 days of exposure at room temperature, $30^{\circ} \mathrm{C}$, and $50{ }^{\circ} \mathrm{C}$ showed that $\mathrm{pLDH}$ proteins were the most temperature labile, and HRP2 and CRP proteins were the least temperature labile across the temperature ranges tested (Online Resource Table 3).

\section{Diagnostic performance of DBS and whole blood against PCR-confirmed infection}

In order to assess the performance of the 5-Plex assay on DBS, the 140 matched DBS and frozen whole blood specimens were analyzed against PCR for sensitivity and specificity. The distribution of biomarker concentration based on PCR-confirmed cases is shown in Fig. 3.

Receiver operating characteristic (ROC) curve analysis was conducted (Online Resource Fig. 3). The area under the curve (AUC) values for Plasmodium antigens are summarized in Table 2. The AUC was highest for HRP2 as a diagnostic marker for $P$. falciparum infection, equally on both DBS $(0.988)$ and whole blood $(0.982)$. The AUCs (0.961, 0.755, and 0.836, respectively) for DBS-based $P f$ $\mathrm{LDH}, \mathrm{P} v \mathrm{LDH}$, and Pan LDH assay were lower than those $(0.969,0.844$, and 0.900 , respectively) for frozen bloodbased assays (Table 2 and Online Resource Fig. 3). The lowest AUC was observed for $P v \mathrm{LDH}$ on DBS and also on whole blood.

Using the same cutoffs that were previously optimized on frozen whole blood targeting a specificity of $\geq 99.5 \%$ against PCR-confirmed infection negative frozen blood samples which were tested by the 5-Plex (Jang et al. 2020), the DBS performed with equivalency in this sample panel with whole blood with the exception of $P v \mathrm{LDH}$, which was significantly less sensitive (Table 3). Using the ROC curve analysis (Online Resource Fig. 3 and Online Resource Table 4), optimized DBS-specific cutoffs resulted in sensitivity and specificity estimates of the 5-Plex with DBS which were similar to those of the 5-Plex with frozen blood (Table 4 and Online Resource Table 4).

\section{Detection of $h r p 2 / 3$ deletions}

The capability of the 5-Plex to identify $P$. falciparum infections that do not express HRP2 was assessed with 

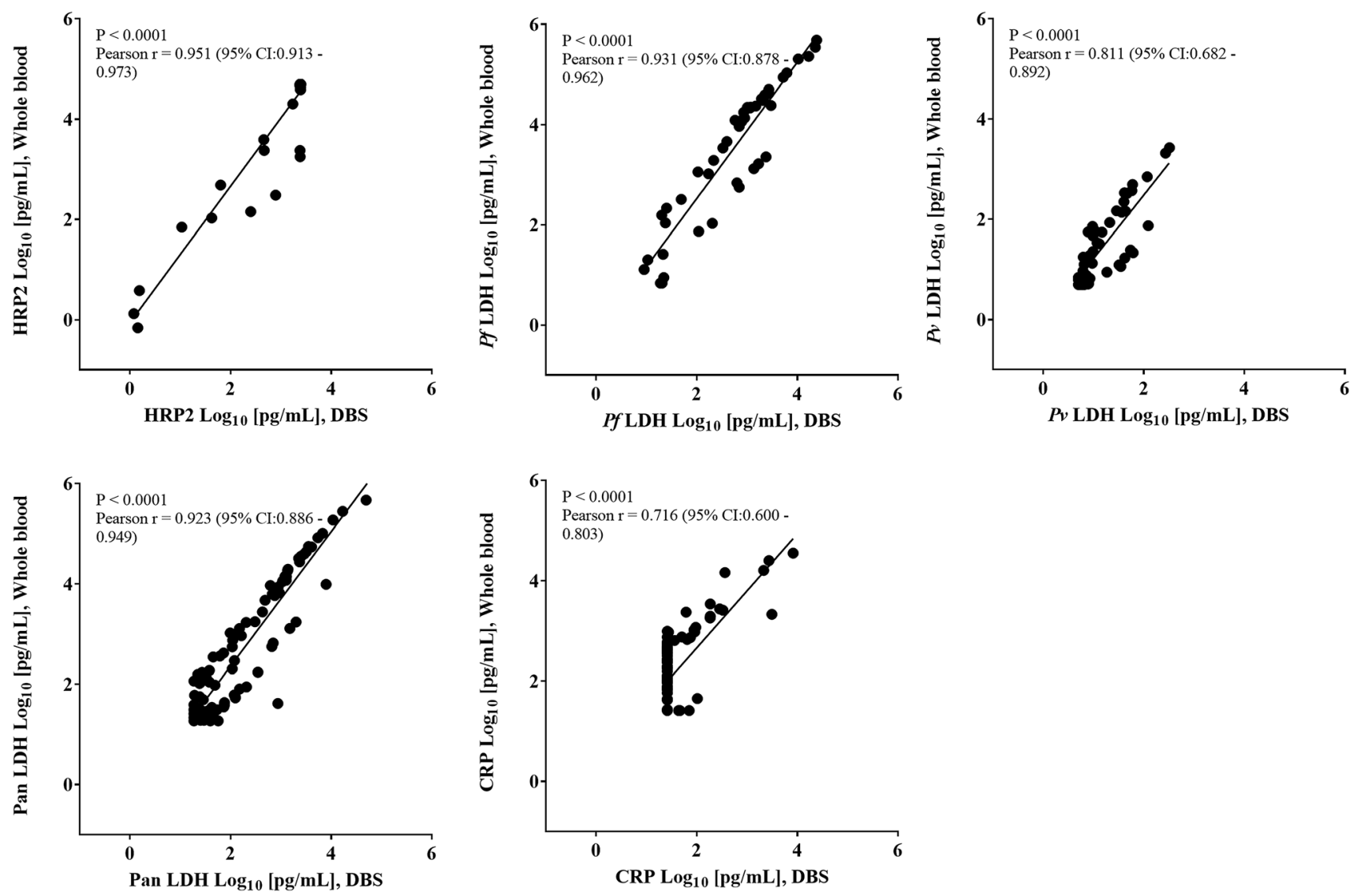

Fig. 1 Correlation in analyte values between DBS and whole blood pellet samples. One hundred forty of the venous blood samples were from two study sites, Uganda and Myanmar, and used to prepare dried blood in the laboratory. Concentration of each analyte from DBS samples (x-axis) was plotted against concentration measured using whole blood pellet samples (y-axis). The dotted line represents the linear trend line for ideal data

respective sample from Study 1, which contained high HRP2 level and parasite density of $10152 \mathrm{p} / \mu \mathrm{L}$.

\section{Discussion}

In a previous study, we described the development and performance characteristics of a commercially available 5-Plex assay (Q-Plex ${ }^{\mathrm{TM}}$ Human Malaria Array) for Plasmodium speciation using frozen whole blood samples (Jang et al. 2020). In this study we further describe the performance of the 5-Plex assay on DBS, a more readily available and convenient sample type for use in low-resource settings. The elution variables on analyte recovery, assay sensitivity and specificity, and thermal stability in DBS were assessed. Optimal elution conditions were explored using contrived DBS samples spiked with HRP2, pLDH, and CRP dried onto Whatman 903 cards (Online Resource Fig. 1). The results revealed that a longer incubation with shaking and increasing larger elution volumes resulted in 

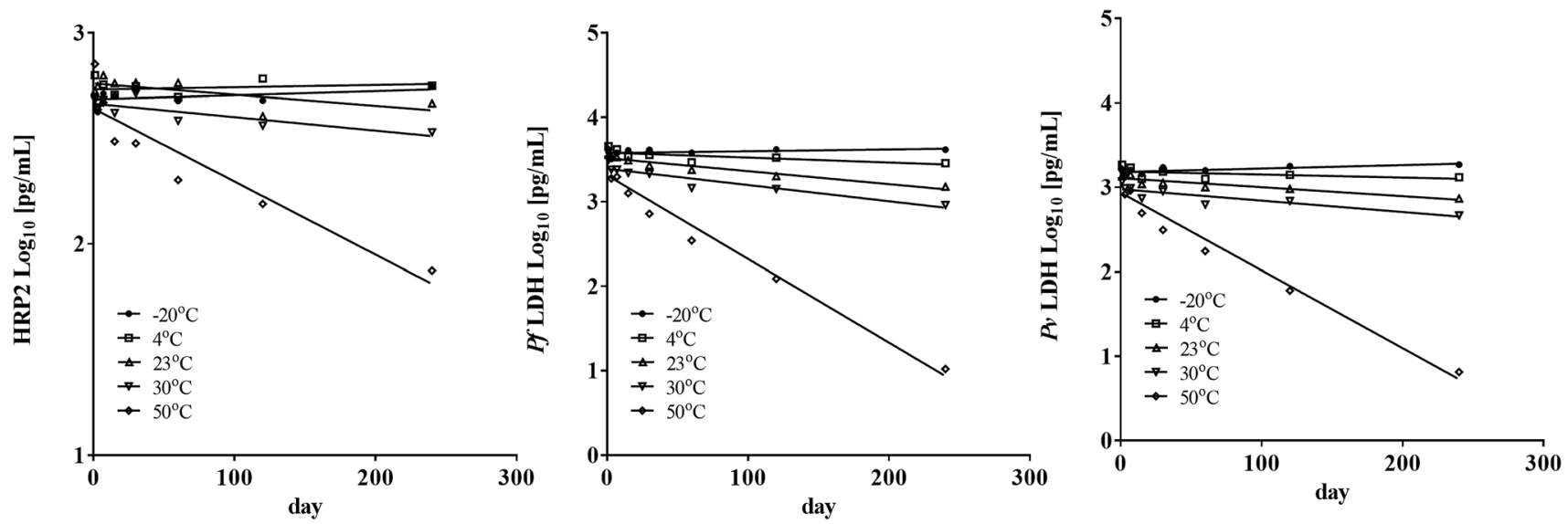

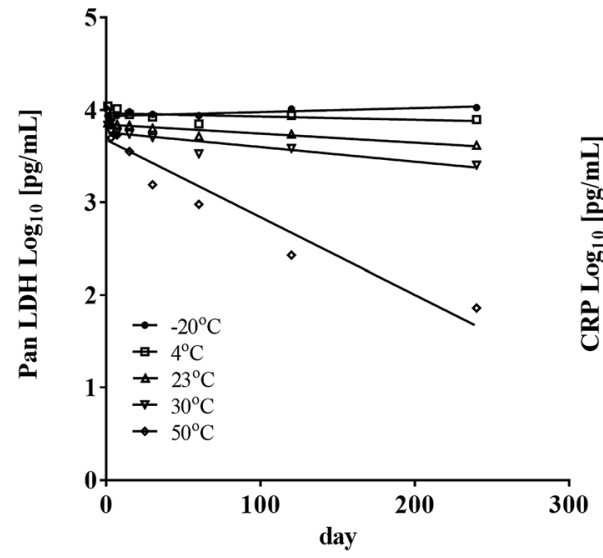

Fig. 2 Analyte concentration recovered from DBS subjected to exposure to different temperatures over time. DBS were tested after storage at various temperature conditions for up to 240 days. The values indicate analyte concentration equivalent to the whole blood pellet sample. The dotted line represents the positive cutoff value for

significant recoveries for the analytes. Additionally, the use of a concentrated competitor mix helped to lower the limit of detection. Using the optimal elution procedure, the recovery of analytes from DBS samples were not equivalent to whole blood pellet samples, indicating the limitation of efficiency of analyte elution from DBS (Online Resource Table 1). Further research efforts are needed to increase the yield allowing efficient recovery from DBS and facilitating the utility of DBS matrices for antigen measurement.

The inter-assay and intra-assay variation experiments on DBS showed acceptable reproducibility for Plasmodium antigens (Table 1) except at the very lower level of analyte where the CV exceeded $20 \%$. The variation observed with inter-assay and intra-assay for CRP were exceeded $20 \%$ and $10 \%$ of $\mathrm{CV}$, respectively. The source of this variation in CRP should be further investigated, taking into consideration that the CRP is measured by competitive ELISA in the 5-Plex assay.
A stability study on Plasmodium antigens in DBS demonstrated that the antigens are better preserved in dried blood samples packaged with desiccants and stored at lower temperature, preferably at $-20{ }^{\circ} \mathrm{C}$ (Fig. 2, and Online Resource Tables 2 and 3). A similar trend has been seen with different analytes in other studies when stored on DBS (Brindle et al. 2019; Gibson et al. 2017). Overall LDH had a consistently shorter half-life than HRP2 at all temperatures including $4{ }^{\circ} \mathrm{C}$, with the exception of $-20^{\circ} \mathrm{C}$. Markwalter et al. (2018) previously reported the loss of both pLDH and HRP2 in DBS by $70 \%$ after 8 days of storage at room temperature. It would be interesting to investigate if the use of native versus recombinant proteins is a reason for this disagreement. The higher temperature lability characteristics of $\mathrm{pLDH}$ has large implications on the use of DBS for screening for infections carrying $P$. falciparum with hrp2/3 deletions, which relies solely on pLDH detection. Our results show that DBS stored for long periods of time may not be reliable for pLDH-based 


\section{HRP2}

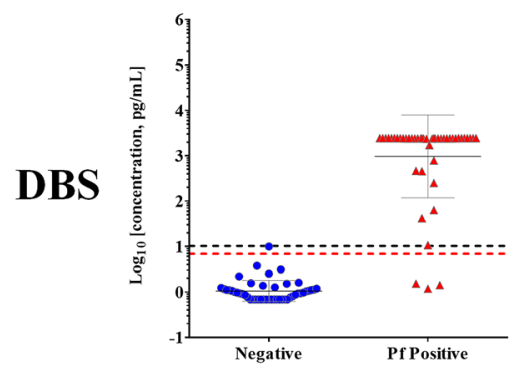

$\operatorname{Pf} \mathrm{LDH}$

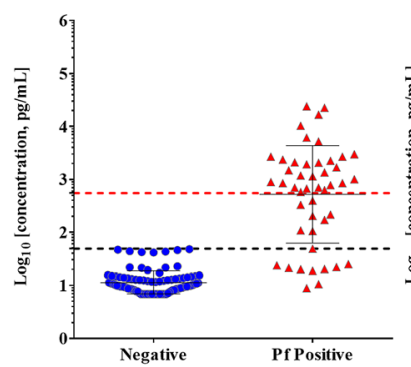

Pv LDH

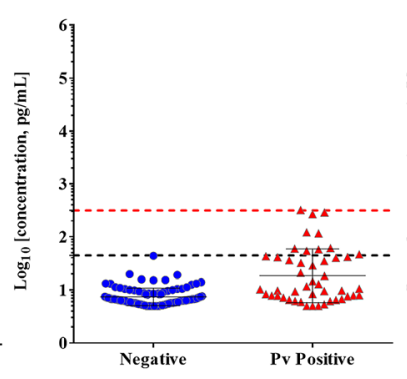

Pan LDH

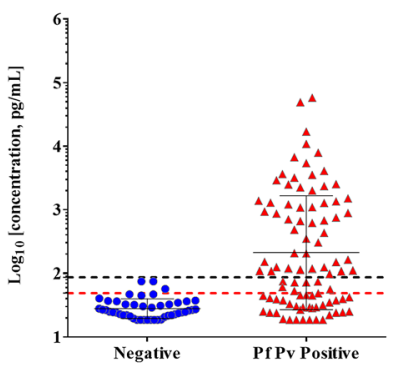

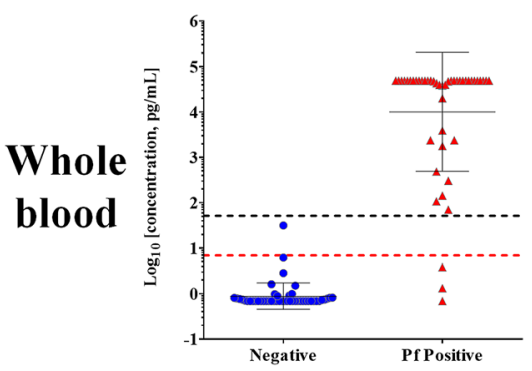

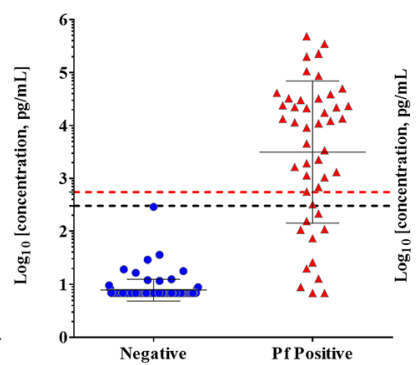

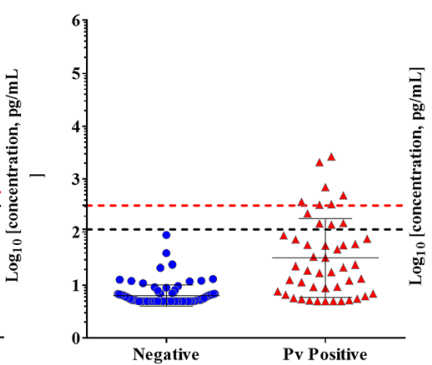

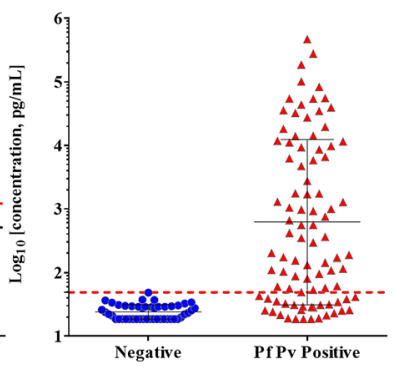

Fig. 3 Distribution of Plasmodium antigens by corresponding PCR results. Scatter dot plots illustrate the log distributions of Plasmodium antigen concentration determined by the 5-Plex as indicated. Parasite positive and negative was determined by PCR. Black dotted line: Cutoff yielding $>99.5 \%$ specificity derived from ROC curve analysis

of data from the 140 samples described here. Red dotted line: Cutoff yielding $>99.5 \%$ specificity derived from ROC curve analysis of data from 462 blood samples described previously (Jang et al. 2020). Two cutoff lines for Pan LDH are located in close proximity

Table 2 Summary of area-under-the-curve (AUC) values derived from the receiver operator characteristics curve analysis (Online Resource Fig. 3) derived from the 140 matched specimens tested in this study both on DBS and whole blood pellets

\begin{tabular}{|c|c|c|c|c|}
\hline & \multicolumn{4}{|c|}{ AUC $(95 \%$ confidence interval) } \\
\hline & HRP2 & $P f \mathrm{LDH}$ & $P v \mathrm{LDH}$ & Pan LDH \\
\hline \multirow[t]{2}{*}{ DBS } & 0.988 & 0.961 & 0.755 & 0.836 \\
\hline & $(0.972-1.004)$ & $(0.924-0.997)$ & $(0.664-0.846)$ & $(0.772-0.901)$ \\
\hline \multirow[t]{2}{*}{ Whole blood } & 0.982 & 0.969 & 0.844 & 0.900 \\
\hline & $(0.950-1.013)$ & $(0.931-1.006)$ & $(0.769-0.919)$ & $(0.851-0.949)$ \\
\hline
\end{tabular}

detection of malaria infection be it hrp2/3 deletions or nonfalciparum infections. Furthermore, DBS should be stored with desiccant at a minimum of $-20{ }^{\circ} \mathrm{C}$ for extended storage. The poor stability of $\mathrm{pLDH}$ imposes the requirement for maintaining good storage conditions and custody for the DBS to ensure reliability of antigen detection assays downstream. While this is also a requirement for the whole blood pellets, typically storage conditions for DBS are not monitored as closely. However, the biomakers are preserved for short period of time if DBS are properly prepared and so may be collected and shipped to a facility for long term cold storage without significant decay.
Clinical samples showed a high correlation between concentration of antigens in matched whole blood and DBS (Fig. 1). For the AUC values obtained from the ROC curve analyses conducted on the HRP2, $P f \mathrm{LDH}, P v \mathrm{LDH}$, and Pan LDH data, those for DBS and whole blood-based HRP2 assays were the highest, indicating that HRP2 assay is very accurate (Table 2). Using PCR results as reference, the sensitivity and specificity of the 5-Plex in diagnosing malaria infection were calculated. Cutoff values previously established for frozen whole blood specimens (Jang et al. 2020) led to the similar sensitivity and specificity estimates for HRP2 assay in DBS and whole blood, but lower sensitivity and specificity estimates for $\mathrm{pLDH}$ assays in DBS 
Table 3 Performance characteristics for each Plasmodium antigen assay used in the 5-Plex HRP2, $P f \mathrm{LDH}$ for $P$. falciparum, $P v \mathrm{LDH}$ for $P$. vivax, and Pan LDH for all Plasmodium species. The cutoff and diagnostic sensitivity/specificity for each assay was assessed in 140 of matched DBS and whole blood pellet samples. The sensitivity and specificity estimates for assays testing the DBS and frozen whole blood specimens in comparison to results of PCR as reference method are shown using cutoffs previously established for frozen whole blood specimens which were tested by the 5-Plex (Jang et al. 2020)

\begin{tabular}{|c|c|c|c|c|}
\hline Target & Cutoff $(\mathrm{pg} / \mathrm{mL})$ & Source & Sensitivity $(95 \% \mathrm{CI})$ & Specificity $(95 \% \mathrm{CI})$ \\
\hline \multirow[t]{2}{*}{ HRP2 } & 6.9 & DBS & $93.5(82.1-98.6)$ & $97.9(88.7-100.0)$ \\
\hline & & Frozen blood & $93.5(82.1-98.6)$ & $97.9(88.7-100.0)$ \\
\hline \multirow[t]{2}{*}{$P f \mathrm{LDH}$} & 553.6 & DBS & $63.0(47.6-76.8)$ & $100.0(96.2-100.0)$ \\
\hline & & Frozen blood & $76.1(61.2-87.4)$ & $100.0(96.2-100.0)$ \\
\hline \multirow[t]{2}{*}{$P v \mathrm{LDH}$} & 314.8 & DBS & $2.1(0.1-11.3)$ & $100.0(96.1-100.0)$ \\
\hline & & Frozen blood & $23.4(12.3-38.0)$ & $96.8(90.9-99.3)$ \\
\hline \multirow[t]{2}{*}{ Pan LDH } & 49.0 & DBS & $63.4(52.8-73.2)$ & $93.6(82.5-98.7)$ \\
\hline & & Frozen blood & $72.0(61.8-80.9)$ & $100.0(92.5-100.0)$ \\
\hline
\end{tabular}

Table 4 Performance characteristics of HRP2, $P f \mathrm{LDH}, P v \mathrm{LDH}$, and Pan LDH assayed on the 5-Plex from DBS. The cutoff and diagnostic sensitivity/specificity for each assay was assessed in 140 of DBS samples using receiver operating characteristic (ROC) analysis. At each cutoff value, sensitivity and specificity of the 5-Plex were calculated with their confidence interval in comparison to PCR results (Online Resource Table 4). The cutoff value to yield a specificity of $99.5 \%$ was selected

\begin{tabular}{ll} 
Sensitivity $(95 \%$ CI $)$ & Specificity $(95 \%$ CI $)$ \\
\hline $93.5(82.1-98.6)$ & $100.0(92.4-100.0)$ \\
$80.4(66.1-90.6)$ & $100.0(96.2-100.0)$ \\
$21.3(10.7-35.7)$ & $100.0(96.1-100.0)$ \\
$55.6(45.2-66.2)$ & $100.0(92.5-100.0)$
\end{tabular}

Table 5 The performance of the 5-Plex to identify $P$. falciparum with $h r p 2 / 3$ deletions in DBS samples collected for two separate studies (Study 1; $\mathrm{n}=19$ and Study 2; $\mathrm{n}=13$ ). For determining \%
Accuracy, $P f$ classification was based on positive results of either $\mathrm{HRP} 2$ or $P f \mathrm{LDH}$, and $h r p 2 / 3$ deletions were reported by detecting the absence and presence of HRP2 and $P f \mathrm{LDH}$, respectively

\begin{tabular}{|c|c|c|c|c|c|c|c|c|}
\hline & \multirow[t]{2}{*}{ Category } & \multirow{2}{*}{$\begin{array}{l}\text { hrp } 2 / 3 \text { Deletions by PCR } \\
\text { Number of samples }\end{array}$} & \multicolumn{4}{|c|}{ 5-Plex, Number of positive samples } & \multicolumn{2}{|c|}{$\%$ Accuracy of 5-Plex } \\
\hline & & & HRP2 & Pf LDH & $P v \mathrm{LDH}$ & Pan LDH & $P f$ classification & hrp2/3 deletions \\
\hline \multirow[t]{4}{*}{ Study 1 (2007-2008) } & Pf hrp $2^{+}$hrp $3^{+}$ & 5 & 5 & 0 & 0 & 5 & 100 & $\mathrm{NA}^{\mathrm{c}}$ \\
\hline & Pf hrp $2^{+}$hrp $3^{-}$ & 6 & 6 & 0 & 0 & 5 & 100 & NA \\
\hline & Pf hrp2 $2^{-}$hrp $3^{+}$ & 1 & 1 & 0 & 0 & 1 & 100 & NA \\
\hline & Pf hrp $2^{-}$hrp $3^{-}$ & 7 & 2 & 0 & 0 & 4 & 28.6 & 0 \\
\hline \multirow[t]{4}{*}{ Study 2 (2014) } & Pf hrp $2^{+}$hrp $3^{+}$ & 2 & 2 & 1 & 0 & 2 & 100 & NA \\
\hline & Pf hrp $2^{+}$hrp $3^{-}$ & 3 & 3 & 3 & 0 & 3 & 100 & NA \\
\hline & Pf hrp $2^{-}$hrp $3^{+}$ & 4 & $0^{\mathrm{a}}$ & 2 & 0 & 4 & 50 & NA \\
\hline & Pf hrp2 $2^{-} h r p 3^{-}$ & 4 & 0 & $3^{\mathrm{b}}$ & 0 & 4 & 75.5 & 75.5 \\
\hline
\end{tabular}

${ }^{\text {a Predicted as }} h r p 2 / 3$ deletions likely due to loss of $h r p 2$, which predominantly express protein

${ }^{\mathrm{b}} \mathrm{A}$ single sample (1/4) failed to detect $P f \mathrm{LDH}$ was reported to have $5252 \mathrm{p} / \mathrm{mL}$ of parasitemia

${ }^{\mathrm{c}} \mathrm{NA}$ : not applicable

(Table 3). These results indicate that there was more difference between DBS and whole blood with regards to the reactivity of $\mathrm{pLDH}$. However, the ROC curve analysis using data from140 DBS samples recommended different cutoff values, but similar sensitivity and specificity estimates especially for HRP2, Pf LDH and Pan LDH assays. Significant limitations of this study could be the relatively small number of matched DBS and frozen blood 
specimens, and the smaller concentration range for each analyte resulted from including only asymptomatic malaria cases from two geographic regions. Consequently, the cutoffs for the 5-Plex using DBS-derived samples tested here may not be optimal and should be further validated with more specimens and from a broader geography.

In addition, our data from a very small sample size suggests that reliable malaria speciation and hrp2/3 deletions screening can be performed on DBS even after at least 4 years storage as long as the DBS have been stored appropriately at $-20{ }^{\circ} \mathrm{C}$ (Table 5). Most of anti-HRP2 antibodies can react to HRP3 which has a sequence homology in the histidine-rich repeat region of HRP2. However, no detectable amount of HRP2 protein found in P. falciparum with $h r p 2^{-} h r p 3^{+}$genotype from Study 2 suggests that the cross-reactivity to HRP3 did not increase the detectability of HRP2 assay due to the lower abundance of HRP3 (Baker et al. 2011).

We previously reported that the increased levels of CRP, an acute phase inflammatory protein are found in febrile malaria cases (Jang et al. 2020). CRP level can predict the risk of complications in febrile malaria infection. Examining the distribution of CRP concentration based on PCR results showed that there was no significant difference between healthy individuals and asymptomatic malariapositive individuals when DBS and whole blood samples were tested (Online resources Fig. 4).

Although these results are preliminary and need more data from a larger sample set to be conclusive, the present study demonstrates that the 5-Plex can utilize DBS as a sample type, increasing the utility of the 5-Plex as a simple and sensitive test to inform on the dynamics of malarial species in low prevalence settings and with the emergence of $P$. falciparum with $h r p 2 / 3$ deletions.

Acknowledgements We greatly acknowledge the support of human volunteers who willingly donated their blood samples for this study. We thank Sophie Knudson and Christine Waresak for their assistance in manuscript proofing and editing. We are grateful to Drs. David Boyle and Allison Golden for their critical reading of the entire manuscript.

Authors' contributions Ihn Kyung Jang and Gonzalo J. Domingo contributed to the design of the study and wrote the manuscript. Sara Aranda, Rebecca Barney, Andrew Rashid, and Muhammad Helwany were responsible for running assay and data analysis. John C. Rek, Emmanuel Arinaitwe, Harriet Adrama, Maxwell Murphy, Mallika Imwong, Stephane Proux, and Warat Haohankhunnatham led the field work; Francois Nosten, Bryan Greenhouse, and Dionicia Gamboa designed the field studies. Xavier C. Ding reviewed and edited this manuscript.

Funding This work was supported by the Bill \& Melinda Gates Foundation (Grant No. OPP1135840). SA and MH were supported by the Siemens Foundation-PATH Fellowship (Grant No. OPP00006234).
Availability of data and material Not Applicable

\section{Compliance with ethical standards}

Conflict of interest All authors declare that they have no conflicts of interest.

Consent to participate Samples were collected under written informed consent from the patient.

Consent for publication Not Applicable.

Code availability Not Applicable.

Ethical approval The study protocol and all steps of practical experiments were approved by the relevant institutional review boards (IRBs).

Open Access This article is licensed under a Creative Commons Attribution 4.0 International License, which permits use, sharing, adaptation, distribution and reproduction in any medium or format, as long as you give appropriate credit to the original author(s) and the source, provide a link to the Creative Commons licence, and indicate if changes were made. The images or other third party material in this article are included in the article's Creative Commons licence, unless indicated otherwise in a credit line to the material. If material is not included in the article's Creative Commons licence and your intended use is not permitted by statutory regulation or exceeds the permitted use, you will need to obtain permission directly from the copyright holder. To view a copy of this licence, visit http://creativecommons.org/licenses/by/4.0/.

\section{References}

Baker J, Gatton ML, Peters J, Ho MF, McCarthy JS, Cheng Q (2011) Transcription and expression of Plasmodium falciparum histidine-rich proteins in different stages and strains: implications for rapid diagnostic tests. PLoS ONE 6:e22593. https://doi.org/10.1371/journal.pone.0022593

Bousema T, Okell L, Felger I, Drakeley C (2014) Asymptomatic malaria infections: detectability, transmissibility and public health relevance. Nat Rev Microbiol 12:833-840. https://doi.org/10.1038/nrmicro3364

Brindle E, Lillis L, Barney R, Bansil P, Lyman C, Boyle DS (2019) Measurement of micronutrient deficiency associated biomarkers in dried blood spots using a multiplexed immunoarray. PLoS ONE

14:e210212. https://doi.org/10.1371/journal.pone.0210212

Chen I, Clarke SE, Gosling R, Hamainza B, Killeen G, Magill A, O'Meara W, Price RN, Riley EM (2016) "Asymptomatic" malaria: a chronic and debilitating infection that should be treated. PLoS Med 13(1):e1001942. https://doi.org/10.1371/journal.pmed.1001942

Das S, Jang IK, Barney B, Peck R et al (2017) Performance of a highsensitivity rapid diagnostic test for plasmodium falciparum malaria in asymptomatic individuals from Uganda and Myanmar and naive human challenge infections. Am J Trop Med Hyg 97(5):1540-1550. https://doi.org/10.4269/ajtmh.17-0245

Das S, Peck RB, Barney R, Jang IK, Kahn M, Zhu M, Domingo GJ (2018) Performance of an ultra-sensitive Plasmodium falciparum HRP2-based rapid diagnostic test with recombinant HRP2, culture parasites, and archived whole blood samples. Malar J 17(1):118. https://doi.org/10.1186/s12936-018-2268-7 
Gamboa D, Ho MF, Bendezu J, Torres K, Chiodini PL, Barnwell JW, Incardona S, Perkins M, Bell D, McCarthy J, Cheng Q (2010) A large proportion of $P$. falciparum isolates in the Amazon region of Peru lack pfhrp2 and pfhrp3: implications for malaria rapid diagnostic tests. PLoS ONE 5:e8091. https://doi.org/10.1371/journal.pone.0008091

Gibson LE, Markwalter CF, Kimmel DW, Mudenda L, Mbambara S, Thuma PE, Wright DW (2017) Plasmodium falciparum HRP2 ELISA for analysis of dried blood spot samples in rural Zambia. Malar J 16:350. https://doi.org/10.1186/s12936-017-1996-4

Goodhew EB, Morgan SM, Switzer AJ, Munoz B, Dize L, Gaydos C, Mkocha H, West SK, Wiegand RE, Lammie PJ, Martin DL (2014) Longitudinal analysis of antibody responses to trachoma antigens before and after mass drug administration. BMC Infect Dis 14:216. https://doi.org/10.1186/1471-2334-14-216

Hurdayal R, Achilonu I, Choveaux D, Coetzer TH, Dean Goldring JP (2010) Anti-peptide antibodies differentiate between plasmodial lactate dehydrogenases. Peptides 31(4):525-532. https://doi.org/10.1016/j.peptides.2010.01.002

Jain P, Chakma B, Patra S, Goswami P (2014) Potential biomarkers and their applications for rapid and reliable detection of malaria. Biomed Res Int 2014:852645. https://doi.org/10.1155/2014/852645

Jang IK, Tyler A, Lyman C et al (2019) Simultaneous quantification of Plasmodium antigens and host factor C-reactive protein in asymptomatic individuals with confirmed malaria by use of a novel multiplex immunoassay. J Clin Microbiol 57(1):e0094818. https://doi.org/10.1128/jcm.00948-18

Jang IK, Tyler A, Lyman C et al (2020) Multiplex human malaria array: quantifying antigens for malaria rapid diagnostics. Am J Trop Med Hyg 102(6):1366-1369. https://doi.org/10.4269/ajtmh.19-0763

Kumar N, Pande V, Bhatt RM, Shah NK, Mishra N, Srivastava B, Valecha N, Anvikar AR (2013) Genetic deletion of HRP2 and HRP3 in Indian Plasmodium falciparum population and false negative malaria rapid diagnostic test. Acta Trop 125(1):119-121.

https://doi.org/10.1016/j.actatropica.2012.09.015

Landier J, Haohankhunnatham W, Das S et al (2018) Operational performance of a Plasmodium falciparum ultrasensitive rapid diagnostic test for detection of asymptomatic infections in eastern Myanmar. J Clin Microbiol. https://doi.org/10.1128/jcm.00565-18

Markwalter CF, Ricks KM, Bitting AL, Mudenda L, Wright DW (2016) Simultaneous capture and sequential detection of two malarial biomarkers on magnetic microparticles. Talanta 161:443-449. https://doi.org/10.1016/j.talanta.2016.08.078

Markwalter CF, Gibson LE, Mudenda L, Kimmel DW, Mbambara S, Thuma PE, Wright DW (2018) Characterization of Plasmodium lactate dehydrogenase and histidine-rich protein 2 clearance patterns via rapid on-bead detection from a single dried blood spot. Am J Trop Med Hyg 98(5):1389-1396. https://doi.org/10.4269/ajtmh.17-0996

Martianez-Vendrell X, Jimenez A, Vasquez A et al (2020) Quantification of malaria antigens PfHRP2 and pLDH by quantitative suspension array technology in whole blood, dried blood spot and plasma. Malar J 19:12. https://doi.org/10.1186/s12936-019-3083-5

Pati P, Dhangadamajhi G, Bal M, Ranjit M (2018) High proportions of pfhrp2 gene deletion and performance of HRP2-based rapid diagnostic test in Plasmodium falciparum field isolates of $\begin{array}{llll}\text { Odisha. Malar } & \text { J } & \end{array}$ https://doi.org/10.1186/s12936-018-2502-3

Pearce RJ, Drakeley C, Chandramohan D, Mosha F, Roper C (2003) Molecular determination of point mutation haplotypes in the dihydrofolate reductase and dihydropteroate synthase of Plasmodium falciparum in three districts of northern Tanzania. Antimicrob Agents Chemother 47(4):1347-1354. https://doi.org/10.1128/aac.47.4.1347-1354.2003

Plucinski MM, Candrinho B, Chambe G et al (2018) Multiplex serology for impact evaluation of bed net distribution on burden of lymphatic filariasis and four species of human malaria in northern Mozambique. PLoS Negl Trop Dis 12(2):e0006278. https://doi.org/10.1371/journal.pntd.0006278

Plucinski MM, Dimbu PR, Fortes F, Murphy SC, Smith NT, Cruz KR, Seilie AM, Halsey ES, Aidoo M, Rogier E (2019) Malaria parasite density in individuals with different rapid diagnostic test results and concentrations of HRP2 antigen. Am J Trop Med Hyg 100(5):1202-1203. https://doi.org/10.4269/ajtmh.19-0006

Rogier E, Plucinski M, Lucchi N, Mace K, Chang M, Lemoine JF, Candrinho B, Colborn J, Dimbu R, Fortes F, Udhayakumar V, Barnwell J (2017) Bead-based immunoassay allows subpicogram detection of histidine-rich protein 2 from Plasmodium falciparum and estimates reliability of malaria rapid diagnostic tests. PLoS ONE 12:e172139. https://doi.org/10.1371/journal.pone.0172139

Slater HC, Ross A, Felger I et al (2019) The temporal dynamics and infectiousness of subpatent Plasmodium falciparum infections in relation to parasite density. Nat Commun 10:1433. https://doi.org/10.1038/s41467-019-09441-1

World Health Organization (WHO) World malaria report 2018. https://www.who.int/malaria/publications/world-malaria-report2018/en/

Wu L, van den Hoogen LL, Slater H, Walker PG, Ghani AC, Drakeley CJ, Okell LC (2015) Comparison of diagnostics for the detection of asymptomatic Plasmodium falciparum infections to inform control and elimination strategies. Nature 528(7580):S86-93. https://doi.org/10.1038/nature16039

Publisher's Note Springer Nature remains neutral with regard to jurisdictional claims in published maps and institutional affiliations. 